\title{
Self-awareness in facial recognition
}

\author{
JOHN H. MUELLER, DOUGLAS R. NICODEMUS, and MICHAEL J. ROSS \\ University of Missouri, Columbia, Missouri 65211
}

\begin{abstract}
Subjects studied facial photographs while comparing them with their actual self, ideal self, or some other person. A subsequent recognition task showed no memory differences due to the study tasks, although in each case photos judged similar were remembered better than those judged dissimilar. There were no apparent face memory differences due to scores on a selfawareness scale.
\end{abstract}

Subjects who are required to make self-comparison decisions about study items generally remember more words judged descriptive of themselves than words judged nondescriptive (e.g., Kuiper \& Rogers, 1979), and retention is generally better following a self-comparison encoding task than after a nonself task (e.g., synonymity judgments or other-person descriptiveness judgments). When the stimuli are faces, the counterpart of descriptiveness is judged similarity to one's face, and similar faces are somewhat better recognized than dissimilar faces. However, the difference between self and other comparisons as orienting tasks is not readily obtained with faces (e.g., Mueller, Courtois, \& Bailis, 1981).

This discrepancy between the results with words and faces may merely reflect some difference in the methodology used in studies of verbal vs. pictorial memory, or it may reflect some more fundamental difference in how self-comparisons affect memory in the two cases. This study was conducted to examine the effect of selfawareness in the context of the self-comparison task for faces. In this regard, Lord (1980) distinguished between two aspects of the self, each having a different value in retention. First, there is the self-schema, a collection of verbal generalizations summarizing the self, and this cognitive framework serves as a very effective mnemonic aid when new information is integrated with it. Second, there is the self-image, our visual representation of ourselves, and this component seems relatively less effective as a memory aid. The difference between the self-image and self-schema is of interest here because self-comparison decisions with faces probably activate the imaginal component, the less effective aspect of self-processing.

In this experiment, we tested the hypothesis that individual differences in knowledge of the self-image might affect the benefits derived from the self-comparison task in face memory. Self-knowledge was assessed by the Buss (1980) Self-Consciousness (SC) Scale. This questionnaire provides separate scores for private SC (related to introspectiveness), public SC (assessing

Reprint requests should be sent to J. H. Mueller, Psychology Department, University of Missouri, Columbia, Missouri 65211. concern with how we appear to others), and social anxiety (measuring how anxious we become in the presence of others). It was expected that high scorers on the private and public scales would benefit more from the self-comparison task than would low scorers because their self-images would be better developed. Some support for this expectation comes from a finding by Lord (1980), using a different self-monitoring scale: High self-monitoring subjects recalled self-referent words better than other-person words.

We further tried to examine self-awareness by an experimental manipulation. Whereas some subjects encoded the target faces with reference to their actual self, which is the usual self-comparison task, other subjects compared the targets with their ideal self-appearance. It seems plausible that these two aspects of the self differ in terms of their development as a function of self-awareness; for example, high self-aware subjects might possess a more detailed ideal self-concept, which would be more useful for encoding new information. Furthermore, the actual and ideal self-images differ in reality status and thus could be differentially effective as study tasks.

\section{METHOD}

\section{Subjects}

Fifty-seven introductory psychology students, 43 women and 14 men, participated as part of course requirements. They were randomly assigned to the actual self, ideal self, or other conditions, 19 per group.

\section{Materials}

Black-and-white slides were made from 80 portraits in college yearbooks, half of men and half of women (all Caucasian). The 80 pictures were divided into two sets of 20 males and 20 females each, each set being counterbalanced as the study and distractor sets.

\section{Procedure}

The study phase was disguised as a rating task, and the impending test was not mentioned. The 40 study slides, 20 of males and 20 of females, were presented one at a time at a 5 -sec rate. Subjects made a judgment about each face and marked the result of their decisions on an answer sheet, using a 1-5 rating scale. Each subject made one of three judgments throughout, with specific instructions provided by a written cover sheet. Subjects rated the extent to which each face "looks like you," 
"looks like you would like to look," or "looks like your favorite television, movie, or sports personality," in the actual self, ideal self, and other conditions, respectively. Subjects were tested in small groups, with each of the three conditions being represented in each session.

The test of incidental learning followed the study phase after about a 3-min delay. The recognition test sequence involved the 40 old target slides and 40 new slides, randomly mixed together and presented one at a time at a 5-sec rate. Subjects indicated whether each slide was old or new by marking an answer sheet. Guessing was not mentioned; no limit was implied on the number of "old" responses allowed, nor were subjects explicitly informed of the number of target slides in the test sequence.

Subjects were debriefed by an experimenter who asked a series of questions concerning the perceived benefits of the study task, anxiety during the experiment, and task difficulty. The other questions will be described in more detail below. Finally, subjects filled out the Buss (1980) Self-Consciousness Scale. This inventory involves 23 items, each answered on a 5-point scale, and it yields three scores: private SC, public SC, and social anxiety.

\section{RESULTS AND DISCUSSION}

Table 1 summarizes the recognition results. For purposes of analysis, items rated 1 or 2 during study were classified as negative (i.e., dissimilar) judgments and items rated 3-5 were labeled as positive (i.e., similar). This pooling was done to obtain a sufficient number of "yes" and "no" choices to include study choice as a two-level repeated-measures factor. For each subject, hit rate was computed separately for "yes" and "no" items, thus adjusting for the different number of each type.

The task main effect was not significant $(p>.05)$ for any of the measures in Table $1 .^{1}$ The choice main effect was significant $[\operatorname{Fs}(1,54)>5.33]$, indicating higher hit rates and d's for "yes" items than for "no" items. The absolute best performance was for the ideal self task, especially for dissimilar targets; however, the Task by Choice interaction was nonsignificant for hit rate and $d^{\prime}[F s(2,54)<2.09]$. These results essentially corroborate earlier findings of a minimal difference between self-comparisons and other "deep" orienting tasks in face recognition (e.g., Mueller et al., 1981).

The ideal and actual self data were pooled, and a median split was performed on the basis of private SC scores. Comparisons of high and low scorers on the various recognition measures failed to reveal any significant differences (ts $<1.07)$. Similar median splits on public SC and social anxiety also failed to reveal perfor-

Table 1

Recognition Performance by Study Task

\begin{tabular}{lcccccccc}
\hline & \multicolumn{3}{c}{ Hit Rate } & & \multicolumn{3}{c}{$\mathrm{d}^{\prime}$} \\
\cline { 2 - 5 } \cline { 7 - 8 } & $\mathrm{T}$ & $\mathrm{Y}$ & $\mathrm{N}$ & Rate & $\mathrm{T}$ & $\mathrm{Y}$ & $\mathrm{N}$ \\
\hline Actual & .71 & .80 & .71 & .22 & & 1.47 & 2.02 & 1.48 \\
Ideal & .75 & .77 & .76 & .19 & & 1.70 & 1.96 & 1.78 \\
Other & .70 & .78 & .67 & .18 & & 1.65 & 2.39 & 1.56 \\
\hline
\end{tabular}

Note-FA = false alarm; $T=$ total, $Y=$ "yes" items, $N=$ "no" items.
Table 2

Correlations Between Self-Consciousness (SC) and Recognition Performance on Positive (P) and Negative (N) Items, Pooling Over Actual and Ideal Self-Comparisons

\begin{tabular}{lrrrrrr}
\hline & \multicolumn{2}{c}{ Hit Rate } & & \multicolumn{2}{c}{$\mathrm{d}^{\prime}$} & \\
\cline { 2 - 3 } & $\mathrm{P}$ & $\mathrm{N}$ & & $\mathrm{P}$ & $\mathrm{N}$ & $\begin{array}{c}\text { False } \\
\text { Alarms }\end{array}$ \\
\hline Private SC & -.06 & -.19 & & .04 & -.03 & -.27 \\
Public SC & .07 & -.10 & & .03 & -.13 & -.05 \\
Social Anxiety & .03 & -.14 & -.06 & -.15 & .12 \\
\hline
\end{tabular}

Table 3

Correlation Between Recognition Performance and Estimates of Facial Characteristics, Pooled Over Actual and Ideal Self Tasks

\begin{tabular}{llllllr}
\hline & \multicolumn{2}{c}{ Hit Rate } & & \multicolumn{2}{c}{$\mathrm{d}^{\prime}$} & \\
\cline { 2 - 3 } \cline { 5 - 6 } & $\mathrm{P}$ & $\mathrm{N}$ & & $\mathrm{P}$ & $\mathrm{N}$ & FA \\
\hline Know Own Face & -.09 & $-.34^{*}$ & -.05 & $-.36^{*}$ & .08 \\
Like Own Face & -.15 & -.08 & -.17 & -.11 & .13 \\
Actual-Ideal Match & -.20 & -.16 & -.08 & -.06 & -.05 \\
Easy to Describe & -.11 & .22 & -.08 & .19 & -.21 \\
Easy to Remember & $-.34^{*}$ & .07 & $-.34^{*}$ & -.05 & .15 \\
Distinctive Face & -.27 & -.01 & -.12 & .06 & -.12 \\
\hline
\end{tabular}

Note $-P=$ positive items, $N=$ negative items; $F A=$ false alarms. $* p<.05$.

mance differences $($ ts $<1)$. This aspect of the data was examined further by computing correlations between the various measures of the Buss (1980) scale and recognition performance. As Table 2 shows, the only correlation showing even marginal significance $(p<.10)$ was between false alarms and private SC.

To explore the possible role of self-knowledge further, the debriefing included asking subjects how well they knew their face, how well they liked their face, how close it was to their ideal appearance, how well they could describe their face, how easy other people found their face to remember, and how distinctive they considered their face to be. All these questions were answered on a $1-5$ scale, 5 being high in each case. The answers were correlated with recognition performance, and the results are shown in Table 3, again pooling over the actual and ideal self conditions. There is nothing to indicate any effect on false alarms, but proclaimed self-knowledge of one's face did have an effect for faces judged dissimilar to one's own, as did proclaimed ease of remembering one's face for target faces judged to be similar. Interestingly, the relationship is inverse; that is, better selfknowledge of one's face led to worse performance on dissimilar targets, and the easier one's face was thought to be to remember, the worse performance became on similar targets.

Although these results seemed fairly clear, we decided to run a fourth group $(n=18)$, using procedures identical to those in the actual self condition, except that the recognition test was administered after a 48 -h delay. It seemed that such a delayed test might be more sensitive to differences in the durability of memory traces due to differences in self-knowledge. These data are shown in 
Table 4

Correlations Between Self-Consciousness (SC) and Recognition Performance on Positive (P) and Negative (N) Items for a 48-h-Delayed Test After Actual-Self Comparisons

\begin{tabular}{|c|c|c|c|c|c|}
\hline & \multicolumn{2}{|c|}{ Hit Rate } & \multicolumn{2}{|c|}{$d^{\prime}$} & \multirow{2}{*}{$\begin{array}{c}\text { False } \\
\text { Alarms }\end{array}$} \\
\hline & $\mathbf{P}$ & $\mathrm{N}$ & $\mathbf{P}$ & $\mathbf{N}$ & \\
\hline Private SC & .08 & .02 & .00 & .23 & -.33 \\
\hline Public SC & .04 & .01 & -.17 & .15 & -.24 \\
\hline Social Anxiety & $-.41 *$ & .22 & -.29 & -.15 & .24 \\
\hline
\end{tabular}

${ }^{*} p<.08$.

Table 4. Again, none of the correlations reached significance, although considering Tables 2 and 4 together reveals a consistent (nonsignificant) tendency for high private SC to lead to fewer false alarms. Apparently, the more one engages in introspective reflection about one's self, the less accepting one is of a distractor face. The marginally significant correlation between hit rate (similar faces) and social anxiety in Table 4 was not apparent in Table 2.

While there is always some hazard in accepting null results, we are inclined to conclude that self-awareness is not a major factor in the effectiveness of the selfcomparison manipulation in face memory. One question that might arise here is the magnitude of the selfawareness difference; that is, did high and low scorers on private SC differ enough for performance effects to emerge? Buss (1980) reviewed a number of studies that obtained effects only when self-awareness was primed in some way, such as by the presence of a small mirror during the experimental session. We are assuming that the study task of performing self-comparisons would induce self-awareness differences in a similarly effective manner. Furthermore, Scheier and Carver (1977) demonstrated that comparable results could be obtained for the mirror manipulation and for the comparison of extreme scorers on the self-consciousness questionnaire.

Of course, Lord (1980) did find a self-monitoring effect for verbal memory, but he used a different questionnaire (one that Scheier and Carver, 1977, reported as uncorrelated to the Buss, 1980 , scale). In addition, Lord's (1980) study likely involved the activation of the propositional self-schema because subjects were tested for verbal recall. It may be that the verbal structure summarizing our self-concept is more clearly differentiated for high self-aware subjects than for less aware subjects, but the differentiation is reduced for the selfimage or is blunted due to the ineffectiveness of the image as a mnemonic. We are inclined to accept Lord's (1980) argument that the self-image is not an effective mnemonic and, thus, self-comparisons have no extraordinary effect on face memory, nor does self-awareness greatly alter the modest value of this encoding system as regards facial memory.

Finally, it must be noted that this conclusion and the present data may appear at variance with results obtained by Yarmey and Johnson (in press): Yarmey and Johnson found that subjects generally showed more false alarms as photographs of themselves increased in judged similarity to the subject's real self. This is the pattern of results generally used to demonstrate the operation of a prototype, in this case a visual representation of one's face. However, we have been concerned with the effect of self-reference on memory for the faces of strangers, not self-recognition. It seems that Yarmey and Johnson's results document the existence of a visual image of our face, but the present results indicate that this selfimage does not have general utility as a structure for processing nonpersonal information. In this regard, the schema corresponding to a self-image is somewhat different from other structures, in that integrating the faces of strangers into the schema as new knowledge does not seem to have the adaptive utility of updating other schemas (including the propositional self-structure). That is, the image of one's own face is perhaps more resistant to updating and, thus, does not function as effectively as a memory aid.

\section{REFERENCES}

Buss, A. H. Self-consciousness and social anxiety. San Francisco, Calif: Freeman, 1980.

KuIPER, N. A., \& Rogers, T. B. Encoding of personal information: Self-other differences. Journal of Personality and Social Psychology, 1979, 37, 499-514.

LoRD, C. G. Schemas and images as memory aids: Two modes of processing social information. Journal of Personality and Social Psychology, 1980, 38, 257-269.

Muelle R, J. H., Courtois, M. R., \& Bailis, K. L. Selfreference in facial recognition. Bulletin of the Psychonomic Society, 1981, 17, 85-88.

Scheier, M. F., \& Carver, C. S. Self-focused attention and the experience of emotion: Attraction, repulsion, elation, and depression. Journal of Personality and Social Psychology, 1977, 35, 625-636.

YARMEY, A. D., \& Johnson, J. Evidence for the self as an imaginal prototype. Journal of Research in Personality, in press.

\section{NOTE}

1. Effects described as significant involve $p<.05$, or better. Effects not discussed failed to reach this level.

(Received for publication June 23, 1981.) 